\title{
Mpviropack Plus: Real Life Study
}

\begin{abstract}
Mohamed Baddar, Taher el Zanaty ${ }^{2}$, Reda Amer ${ }^{2}$, Mohsen Salama ${ }^{5}$, Ramy M Elsharkawy ${ }^{6}$, Hazem Elbedawei ${ }^{7}$, Alaa Osman Elroby ${ }^{8}$, Osama A Sabry ${ }^{9}$, Tamer $\mathbf{G}^{10}$, Hares $\mathbf{M}^{11}$, Ayman El Badawy ${ }^{13}$ Nader A Nemr ${ }^{12}$, Mohamed M Aamer ${ }^{14}$, Nashaat Hawass ${ }^{12}$, George Marzouk ${ }^{15}$, Sherif Ragheb ${ }^{15}$, Mohamed B Hashem*1 and Esmat $G^{1}$
\end{abstract}

${ }^{1}$ Endemic medicine department, Cairo University, Egypt

${ }^{2}$ Internal medicine department, Cairo University, Egypt

${ }^{3}$ Internal medicine department, National Hepatology and Tropical Medicine Research Institute, Egypt

${ }^{4}$ Ain Shams University, Egypt

${ }^{5}$ Hepatology Institute, Menoufia, Egypt

${ }^{6}$ Gastroenterology department, Sohag University, Egypt

${ }^{7}$ Medical insurance center, Tanta, Egypt

${ }^{8}$ Gastroenterology and Hepatology department, El fayoum el aam hospital, Egypt

${ }^{9}$ Internal medicine department, Zagazig hospital, Egypt

${ }^{10}$ Ain Shams specialized hospital, Damietta, Egypt

${ }^{11}$ Kafr Saad hospital, Damietta, Egypt

${ }^{12}$ Endemic and Infectious Diseases Faculty of Medicine Suez Canal University, Egypt

${ }^{13}$ Internal medicine department, Banha University, Egypt

${ }^{14}$ Shebin El- Kom teaching hospital, Egypt

${ }^{15}$ Marcyrl Ph Ind, Egypt

Received: March 12, 2018; Published: April 05, 2018

*Corresponding author: Mohamed B Hashem, Endemic medicine department, Cairo University, Giza, Egypt, Email: mohamedhashem50@hotmail.com

\begin{abstract}
Abbreviations: WHO: World Health Organization; PegIFN: Pegylated Interferon; DAAs: Direct Acting Antiviral Drugs Daas; AST: Aspartate Transaminase; ALT: Alanine Transaminase; ALB: Albumin; INR: International Normalized Ratio; CBC: Complete Blood Count; AFP: Alpha Feto Protein; FBS: Fasting Blood Sugar; SVR: Sustained Virological Response
\end{abstract}

\section{Introduction}

Egypt has the highest prevalence of HCV worldwide. It is estimated to be $7.3 \%$ which is considered more than double the global prevalence that is estimated to be $3 \%$ according to the World Health Organization (WHO). The main genotype in Egypt is genotype 4 [1]. For many years ago, HCV treatment remained a challenge, where the available regimens were the moderately potent but poorly tolerated combination of pegylated interferon (PegIFN) and ribavirin for 24 or 48 weeks [2]. Using this combination, HCV genotype 4 patients had intermediate SVR rates (50-60\%) [3]. Since 2011, multiple new drugs acting on specific enzymatic sites throughout the HCV life cycle were developed (direct acting antiviral drugs DAAs). DAAs are more potent than the INF/RBV with bettersafety profile. DAAs are now widely available worldwide [4].

Many interferon-free regimens for treatment of HCV genotype 4 infections are available [5]. One of those regimens includes the use of NS5B polymerase inhibitor Sofosbuvir along with the NS5A inhibitor Ledipasvir. Sofosbuvir and ledipasvir are available in a two-drug fixed-dose combination containing $400 \mathrm{mg}$ of sofosbuvir and $90 \mathrm{mg}$ of ledipasvir in a single tablet. This combination is taken as one tablet orally once daily with or without food [6]. Many clinical trials were done on the combination of sofosbuvir and ledipasvir in HCV genotype 4 patients such as the Synergy trial. SVR12 rates in those studies ranged from $91 \%$ to $100 \%$ depending 
on fibrosis stages and previous treatment experience [6]. However, the relatively high price of the brand drug remained a challenge as many Egyptian HCV patients had financial difficulty in obtaining the drug. This drew our attention to study the safety and efficacy of a generic product of Sofosbuvir/Ledipasvir as this may represent a clue for mass treatment of Egyptian patients. The aim of this study was to assess the safety and efficacy of the generic products of sofosbuvir/Ledipasvir in treating HCV genotype 4 Egyptian patients.

\section{Patients and Methods}

In this study, six hundred and forty nine Egyptian patients chronically infected with HCV genotype 4 were enrolled. The work was carried out in accordance with Helsinki declaration, and following the guidelines of the ethical approval committees in Egypt. Patients were included according to the approved treatment recommendations by EASL 2016 [6]. All patients were subjected to full history taking including history of other chronic liver disease, and laboratory investigations including: Liver biochemical profile (total and direct Bilirubin, Aspartate transaminase (AST), Alanine transaminase (ALT), Albumin (ALB), International normalized ratio (INR). Also complete blood count (CBC), HBsAg, HCV PCR quantitative, serum Creatinine, Alpha-feto protein (AFP), and fasting blood sugar (FBS), HbA1c if diabetic. Pregnancy test was done for female patients in childbearing period. Fib-4 index was calculated to all patients using the formula: age (years) $\mathrm{X}$ AST [U/L] / (platelets [109/L] X (ALT [U/L])1/2 [7]. Abdominal ultrasonography was done to each patient to detect; echo-pattern of the cirrhosis, signs of portal hypertension, and to exclude hepatocellular carcinoma. Patients were then categorized into easy (326 patients) and difficult-to-treat (323 patients) groups. Those considered difficult to treat were excluded from the study. The easy to treat group included noncirrhotic patients (by clinical \& ultrasonographic examination), Fib-4 $<3.25$, albumin $>3.5$, total serum Bilirubin $<1.2 \mathrm{mg} / \mathrm{dL}, \quad$ INR $<1.2$ and Platelet count $>=150000 \mathrm{~mm} 3$, while difficult-to-treat group included those who were cirrhotic (by clinical \& ultrasonographic examination), Fib$4>3.25$, albumin $\leq 3.5$, total serum bilirubin $>1.2 \mathrm{mg} / \mathrm{dL}$, INR $>1.2$, and platelet count $<150000 \mathrm{~mm} 3$ [8].

\section{Treatment Regimen}

All patients received Sofosbuvir 400mg/Ledipasvir 90mg (Mpviropack plus) without Ribavirin for 12 weeks according to EASL guidelines [2]. The end point was a sustained virological response at 12 (SVR12) weeks post treatment. During the period of HCV treatment, concurrent treatments given for comorbid conditions that might affect the efficacy or may increase the toxicity of this regimen were revised and whenever possible were stopped or switched to alternative ones with less interaction potentials.

\section{Monitoring of Treatment Safety}

While on treatment, patients were followed up every 4 weeks and for 12 weeks post treatment (to test for SVR12). During treatment period, any adverse event were documented and managed accordingly. Patients were asked specifically about the commonly reported effects reported with the brand drug as headache, nausea, insomnia, fatigue, rash, dyspnea, etc.

\section{Monitoring of Treatment Efficacy}

Quantitative HCV-PCR was measured using the COBAS Ampliprep/COBAS TaqMan (CAP/CTM) assay with a lower limit of detection of $15 \mathrm{IU}$ prior to treatment, and 12 weeks after treatment. Virologic response was considered when HCV RNA was less than the lower limit of detection at week 12 post treatment (SVR12). Treatment failure was defined as confirmed HCV RNA above LLOQ 12 weeks post treatment. Treatment discontinuation due to adverse events was considered treatment failure.

\section{Statistical Analysis}

Data analysis was done using Statistics/Data Analysis (STATA) version 13.1 software. Continuous variables were tested for normality by the Shapiro-Wilk normality test. Values are presented as mean \pm standard deviation, or in the case of non-normally distributed data as median and inter-quartile range. Normally distributed paired samples were analyzed using the paired t-test. Non-normally distributed paired samples were analyzed using the Wilcoxon signed-rank test.

\section{Results}

This study included an easy to treat 326 Egyptian patients with HCV Genotype 4 infection. Most of patients were males (61.96\%), mean age was 46.84 years, all were non-cirrhotics by laboratory and ultrasonographic evidence as well as a Fib-4 index $<3.25$. All of them were treatment naïve, $42(12.88 \%)$ patients had history of controlled diabetes mellitus. Mean albumin level was 3.95 while mean platelet count was 189,650 (Table 1). All patients in the current study received the fixed dose combination sofosbuvir/ ledipasvir without ribavirin for 12 weeks (Table 2).

Table 1: Demographic and Clinical Characteristics of the Studied Population. (Sofosbuvir/Ledipasvir for 12 weeks, $n=326$ ).

\begin{tabular}{|c|c|c|}
\hline \multicolumn{2}{|c|}{$\begin{array}{c}\text { Demographic and Clinical Characteristics of } \\
\text { the Studied Population }\end{array}$} & \multirow{2}{*}{$\begin{array}{c}\text { Number } 326(\%) \\
202(61.96 \%)\end{array}$} \\
\hline \multirow{2}{*}{ Gender } & Male & \\
\hline & Female & $124(38.04 \%)$ \\
\hline \multicolumn{2}{|c|}{ Non-cirrhotics } & $326(100 \%)$ \\
\hline \multicolumn{2}{|c|}{ Treatment naïve } & $326(100 \%)$ \\
\hline \multicolumn{2}{|c|}{ Diabetes Mellitus } & $42(12.88 \%)$ \\
\hline & & Mean (SD) \\
\hline \multicolumn{2}{|c|}{ Age } & $46.84 \pm(2.4)$ \\
\hline \multicolumn{2}{|c|}{ Albumin (g/dL) } & $3.95 \pm(0.56)$ \\
\hline \multicolumn{2}{|c|}{ Platelet count $\left(\mathrm{x} 10^{3} \mathrm{~mm}^{3}\right)$} & $189.65 \pm(27.2)$ \\
\hline
\end{tabular}

Table 2: Svr12 Rate among Studied Population. All But 4 of the Studied Patients Had Svr12 (98.77\%)Minor Side Effects Were Observed In the Form of Fatigue (30\%), Headache (24\%), Nausea (15\%) and Insomnia (11\%).

\begin{tabular}{|c|c|}
\hline SVR12 & Number (\%) \\
\hline Yes & $322(98.77 \%)$ \\
\hline No & $4(1.23 \%)$ \\
\hline
\end{tabular}




\section{Discussion}

Elimination of HCV in Egypt was a target since long time, however, the absence of effective and safe antiviral treatment remained a challenges towards achieving this target. Recently, introduction of the new oral directly acting antiviral drugs (DAAS) in the national treatment program in Egypt carried a hope towards achieving the goal of HCV elimination, However, still the price of the brand products represent a challenging difficulty as Egypt is a country with low gross national income per capita. Although, the development of generic products of the DAAS carried a solution for this obstacle, yet, data available on the safety and efficacy of these generic products were very limited compared to the brand ones. In this study, drugs used were very effective with SVR12 rate $98.77 \%$. These results were superior to the only two published studies done on HCV genotype 4 patients where in the Synergy trial, 21 patients were included with SVR12 rate 95\% [9] while in other study that included 22 naive patients showed an SVR12 rate of 96\% [10] . In the same study, the fixed dose combination of sofosbuvir/ledipasvir was assessed in treating 22 experienced patients showing SVR12 rate of $91 \%$. However, the exclusion of treatment experienced patients in our study makes it inappropriate comparison [10]. The side effects of the generic drugs used, was nearly the same known for the brand ones.

\section{Conclusion}

HCV elimination in Egypt is a nation-wide target with an expected positively rewarding impact on both the public health as well as reduction on the economic burden. An available generic product having almost the same efficacy and safety of the brand ones but with a much lower cost, will allow the mass treatment of chronic HCV Egyptian patients. This makes the dream of HCV elimination in Egypt an achievable goal.

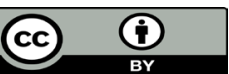

This work is licensed under Creative Commons Attribution 4.0 License

Submission Link: https://biomedres.us/submit-manuscript.php

\section{References}

1. Waked I, Doss W, El Sayed M, Estes C, Razavi H, et al. (2014) the current and future disease burden of chronic hepatitis $C$ virus infection in Egypt. Arab J Gastroenterol 15(2): 45-52.

2. (2011) European Association for the Study of the Liver. EASL Clinical Practice Guidelines: management of hepatitis $C$ virus infection. J Hepatol 55: 245-264.

3. Antaki N, Craxi A, Kamal S, Moucari R, Van der Merwe S, et al. (2010) The neglected hepatitis $C$ virus genotypes 4,5 , and 6: an international consensus report. Liver Int 30: 342-355.

4. Poordad F, Dieterich D (2012) Treating hepatitis C: current standard of care and emerging direct-acting antiviral agents. J Viral Hepat 19: 449464.

5. Abdel Razek, W Waked I (2015) Optimal therapy in genotype 4 chronic hepatitis C: finally cured? Liver Int 35 Suppl 1: 27-34.

6. J Hepatol (2016) EASL Recommendations on Treatment of Hepatitis C.

7. Sterling RK, Lissen E, Clumeck N, Sola R, Correa MC, et al. (2006) Development of a simple noninvasive index to predict significant fibrosis in patients with HIV/HCV coinfection. HEPATOLOGY 43: 1317-1325.

8. Guerra J, Garenne M, Mohamed MK, Fontanet A (2012) HCV burden of infection in Egypt: results from a nationwide survey. Journal of Viral Hepatitis 19: 560-567.

9. Kapoor R, Kohli A, Sidharthan S, Sims Z, Petersen TL, et al. (2014) All oral treatment for genotype 4 chronic hepatitis $C$ infection with sofosbuvir and ledipasvir: interim results from the NIAID SYNERGY trial. Hepatology 60: 321A.

10. Abergel A, Metivier S, Samuel D, Jiang D, Kersey K, et al. (2016) Ledipasvir plus sofosbuvir for 12 weeks in patients with hepatitis $\mathrm{C}$ genotype 4 infection. Hepatology 64(4): 1049-1056.

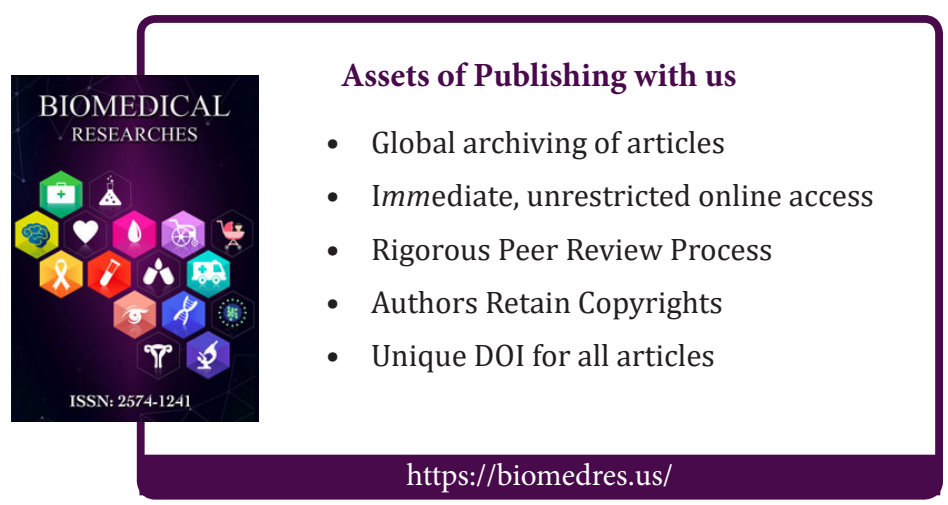

